\title{
MiR-376c-3p targets heparin-binding EGF-like growth factor (HBEGF) to inhibit proliferation and invasion in medullary thyroid carcinoma cells
}

\author{
Ning Bai ${ }^{1,2}$, DeQiang $\mathrm{Hou}^{2}$, ChunPu Mao ${ }^{2}$, Liang Cheng ${ }^{3}$, $\mathrm{Na} \mathrm{Li}^{1}$, XiaoMing Mao ${ }^{1}$
}

\begin{abstract}
1Department of Endocrinology, Nanjing First Hospital, Nanjing Medical University, Nanjing, China

2Department of Endocrinology, Affiliated Hospital of Jiangnan University, Jiangsu, China

${ }^{3}$ Department of Endocrinology, Huai'an Second People's Hospital, Huai'an, China
\end{abstract}

Submitted: 10 September 2018

Accepted: 3 January 2019

Arch Med Sci 2020; 16 (4): 878-887

DOI: https://doi.org/10.5114/aoms.2019.85244

Copyright @ 2019 Termedia \& Banach

\begin{abstract}
Introduction: Aggressive medullary thyroid carcinomas (MTC) have a high mortality rate and the treatment for patients diagnosed with advanced MTC is comparatively ineffective. We hence aimed to test the effects of miR-376c-3p on MTC and to explore the relevant mechanism.

Material and methods: Cell Counting Kit- 8 (CCK-8) and soft agar colony formation assay were applied to evaluate the proliferation of transfected MZ-CRC-1 cells. Wound healing and transwell assay were employed to evaluate MTC cell migration and invasion, respectively. Luciferase assay was performed to validate the downstream target of miR-376c-3p in MZ-CRC-1 cells. Quantitative polymerase chain reaction was used to detect mRNA abundance of key genes. Western blot technique was used to analyze protein levels of HBEGF, E-cadherin, ZO-1, N-cadherin and vimentin.

Results: MiR-376c-3p inhibited the viability, migration and invasion of MZ-CRC-1 cells. Moreover, miR-376c-3p mimic downregulated expression of $\mathrm{N}$-cadherin and vimentin but upregulated that of E-cadherin and ZO-1 in MZ-CRC-1 cells. Results for the luciferase reporter assay showed that miR-376c-3p was able to bind the $3^{\prime}$ untranslated region of heparin-binding EGF-like growth factor (HBEGF), of which overexpression nearly nullified the miR-376c-3p mimic-induced inhibitory effects in the MTC cells.

Conclusions: MiR-376c-3p showed suppressive effects on MZ-CRC-1 cells via targeting and downregulating HBEGF, suggesting that miR-376c-3p could potentially be targeted for the treatment of MTC.
\end{abstract}

Key words: miR-376c-3p, medullary thyroid carcinomas, heparin-binding EGF-like growth factor, mechanism, MZ-CRC-1.

\section{Introduction}

Medullary thyroid carcinoma (MTC) is a type of neuroendocrine tumor that originates from thyroid parafollicular $C$ cells. It is also the third most common thyroid cancer, making up $3 \%$ of all cases of thyroid tumors [1, 2]. Current approaches to treat MTC rely mainly on surgical removal [3], the outcome of which makes the 10-year survival rate of the patients $95 \%$. Treatments for late-stage MTC are proved to be comparatively ineffective
Corresponding author:

XiaoMing Mao

Department of Endocrinology

Nanjing First Hospital

Nanjing Medical University

68 Changle Road

Nanjing, Jiangsu, China

Phone: +86 2552887071

E-mail: XiaoMingMaozxc@163.com 
$[4,5]$ with a remarkable drop in the 10-year survival rate to around $55 \%$, especially for the MTC patients with distant metastases. It would hence be useful to explore the underlying mechanisms for MTC progression and metastasis in order to develop new therapeutic methods.

MiRNAs are a family of small non-coding RNAs consisting of approximately 22 nucleotides. They bind the $3^{\prime}$ untranslated region (3'UTR) of target mRNAs at the seeding regions, subsequently leading to the degradation or silencing of the target mRNAs $[6,7]$. It is generally believed that abnormal expression of miRNAs is involved in the initiation, development and metastasis of various tumors: Li et al. reported that miR-34a repressed the metastasis of hepatocellular carcinoma in vitro [8]; Ye et al. demonstrated that miR-139 restricted the development of papillary thyroid carcinoma in vitro [9]; Cheng et al. showed that miR-150 suppressed the growth, migration and invasion of papillary thyroid carcinoma cells [10].

Recent studies reported that miR-376c-3p exhibited protective behaviors against various tumors. For example, Zhang et al. reported that miR$376 c-3 p$ reduced colorectal cancer cell viability and induced apoptosis [11]; Chang et al. reported that down-regulation of miR-376c-3p stimulated lymph node metastasis in head and neck squamous cell carcinoma (HNSCC) [12]; Wang et al. demonstrated that miR-376c-3p downregulation promoted the cancer phenotype of oral squamous cancer cells in vitro [13]. However, the effects of miR-376c-3p on the growth and metastasis of MTC have not been fully understood.

In the present study, the comprehensive effects of miR-376c-3p regarding the proliferation, migration and invasion of the human MTC cell line MZ-CRC-1 was examined using the Cell Counting Kit-8 (CCK-8), soft agar colony formation, wound healing and transwell assay. Dual luciferase reporter assay validated that miR-376c-3p bound 3'UTR of heparin-binding EGF-like growth factor (HBEGF). It was also found that the expression of miR-376c-3p was inversely proportional to that of HBEGF in MTC cells. A possible molecular mechanism for miR-376c-3p protection against MTC was also proposed. In conclusion, miR-376c-3p showed suppressive effects on MZCRC-1 cells via targeting HBEGF and further inhibiting proliferation, migration and invasion of MTC.

\section{Material and methods}

Cell culture

The MZ-CRC-1 cell line, one of the mutant cell lines rearranged during transfection (RET), was acquired from the Cancer Research Institute of Beijing (China). Cells were maintained in high-glucose DMEM medium (Life Technologies, Shanghai, China) supplemented with 10\% FBS (Gibco, Grand
Island, NY) and penicillin-streptomycin (Invitrogen) at $37^{\circ} \mathrm{C}$ within a humidified atmosphere containing $5 \% \mathrm{CO}_{2}$.

\section{MiRNAs and transfection}

MiR-376c-3p mimic, miR-376c-3p inhibitor and corresponding negative controls (NC mimic or NC inhibitor) were designed and synthesized by GenePharma (Shanghai, China). Cell transfection was performed with Lip2000 (Invitrogen Life Technologies, Shanghai, China).

\section{CCK-8 assay}

CCK-8 assay was performed following procedures that have been previously described [14]. Transfected MZ-CRC-1 cells (2,000 cells per well) were seeded into 24-well plates and incubated for 0, 24, 48 or 72 h. CCK-8 (Dojindo, Shanghai, China) was added into each well and incubated for $3 \mathrm{~h}$, after which the absorbance was read at a wavelength of $450 \mathrm{~nm}$.

\section{Soft agar colony formation assay}

MZ-CRC- 1 cells (1000) were suspended in $0.35 \%$ agar gel containing 2\% FBS and then transferred to $0.6 \%$ agar gel containing $2 \%$ FBS in 6-well plates. After 3 weeks, cells were fixed with formaldehyde and dyed with crystal violet. The colonies were then counted.

\section{Wound healing assay}

Cells were incubated in 6-well plates for $24 \mathrm{~h}$ and then wounded with a $200-\mu l$ pipette tip. The wounded cells were incubated for another $24 \mathrm{~h}$ and the cell migration images were captured and the wound width was examined afterwards.

\section{Transwell assay}

Transfected cells were collected and suspended in serum-free medium and were then transferred into the upper chamber precoated with Matrigel. Medium containing 10\% FBS was added into the lower chamber. After $48 \mathrm{~h}$, cells remaining in the upper chamber were removed and those that had passed through the membrane were fixed with paraformaldehyde and dyed with $0.1 \%$ crystal violet. The stained cells were photographed and counted under an inverted microscope.

\section{Dual luciferase reporter assay}

HBEGF 3'UTR that possessed the putative miR$376 c-3 p$ binding sequences was synthesized and inserted into pGL4 (Promega, Madison, WI, USA) to construct the wild-type plasmid (HBEGF 3'UTR-WT). The mutant HBEGF $3^{\prime}$ UTR possessing the mutant 
putative binding sites of miR-376c-3p was amplified to generate the mutant report plasmid (HBEGF 3'UTR-Mut). MZ-CRC-1 cells were co-transfected with miR-376c-3p mimic or NC mimic and HBEGF 3'UTR-WT or HBEGF 3'UTR-Mut. Luciferase reporter assay was performed after $48 \mathrm{~h}$ of incubation using the dual luciferase assay system (Promega, USA).

\section{Quantitative PCR (qPCR)}

Total RNA was extracted from the MTC cells and was then reverse transcribed using a cDNA reverse transcription kit (Takara, Beijing, China). Bestar real time PCR Master Mix (TaKaRa, Dalian, China) was used to examine the abundances of miR-376c-3p and HBEGF mRNA. QPCR reactions were carried out using an ABI7500 System (Applied Biosystems, USA). Relative mRNA levels of miR-376c-3p and HBEGF were calculated using the $2^{-\Delta \Delta c t}$ method. Primer sequences are listed below:

HBEGF-fwd: 5'-CTGTCTTCGGTCTGCCTCCTT-3', HBEGF-rev: 5'-AGACCGAAGACAGC ACCAC-3', miR-376c-3p-RT: 5'-GTCGTATCCAGTGCAGGGTCCGAGGTATTCGCACTGGATACGACACGTGG-3', miR-376c-3p-fwd: 5'-ACACTCCAGCTGGGAACATAGAGGAAATT-3',

miR-376c-3p-rev: 5'-GTGCAGGGTCCGAGGT-3'.

\section{Western blot}

Western blot was performed following procedures that have been previously described [15]. Primary polyclonal antibodies targeting E-cadherin, ZO-1, N-cadherin, vimentin and HBEGF were bought from Abcam. Cells were lysed and precipitated by centrifugation, followed by the extraction and separation of proteins. The extracted proteins were transferred onto PVDF membranes (Pharmacia Corporation, NJ, USA) and blocked. Membranes were then probed with primary polyclonal antibodies. Primary antibodies that bound the target proteins were analyzed using the corresponding secondary antibodies (1 : 500; Promega).

\section{Statistical analysis}

Data collected from three independent experiments were processed using SPSS 12.0 software and reported as mean \pm SEM. Values of $p<0.05$ were considered to be statistically significant.

\section{Results}

\section{MiR-376c-3p overexpression inhibited MTC cell proliferation}

MZ-CRC-1 cells were transfected with miR-376c-3p mimic or miR-376c-3p inhibitor. MTC cells transfected with the miR-376c-3p mimic showed a higher expression level of miR-376c-3p mRNA than those transfected with the NC mimic $(p<0.001)$ (Figure $1 \mathrm{~A})$.
In contrast, cells transfected with the miR-376c-3p inhibitor showed a lower expression level of miR376c-3p mRNA, compared to those transfected with the NC inhibitor $(p<0.01)$ (Figure $1 \mathrm{~A}$ ).

CCK-8 assay results (Figure 1 B) showed decreased OD values of the miR-376c-3p mimic group compared with the NC mimic group at the time points of $24(p<0.05), 48(p<0.001)$ and $72 \mathrm{~h}(p<0.001)$, whereas increased OD values were observed in the miR-376c-3p inhibitor group compared with the NC inhibitor group at the $48^{\text {th }}(p<0.05)$ and $72^{\text {nd }} \mathrm{h}(p<0.01)$. In addition, miR-376c-3p mimic reduced colony formation of MTC cells to $20 \%$ compared with the NC mimic $(p<0.01$ ) (Figure $1 \mathrm{C}$ ), while the miR-376c-3p inhibitor significantly induced the colony formation of MTC cells compared with the NC inhibitor $(p<0.05)$ (Figure $1 \mathrm{C}$ ).

\section{MiR-376c-3p overexpression inhibited migration and invasion of MTC cells}

Results of the wound healing assay showed that the migratory ability of MZ-CRC-1 cells of the miR-376c-3p mimic group was dramatically limited compared to that of cells of the NC mimic group $(p<0.01)$ (Figure $2 \mathrm{~A})$. In contrast, miR-376c-3p inhibitor enhanced the migratory ability of MTC cells $(p<0.05)$ (Figure $2 \mathrm{~A})$. The transwell assay showed that the miR-376c-3p mimic significantly inhibited MTC cell invasion, whereas miR-376c-3p inhibitor promoted it $(p<0.05)$ (Figure 2 B). Additionally, MZ-CRC-1 cells exhibited a change in epithelial morphology when miR-376c-3p was upregulated, whereas downregulation of miR-376c-3p promoted the cell invasion phenotype (Figure $2 \mathrm{C}$ ).

Next, expression level of epithelial-mesenchymal transition (EMT) markers (including E-cadherin, ZO-1, N-cadherin and vimentin) was examined. MiR-376c-3p overexpression inhibited expression of $\mathrm{N}$-cadherin $(p<0.05)$ and vimentin $(p<0.01)$ but induced expression of E-cadherin $(p<0.01)$ and ZO-1 ( $p<0.01)$ in MZ-CRC-1 cells (Figure $2 \mathrm{D}$ ). In contrast, reduced expression of miR-376c-3p dramatically upregulated expression of $\mathrm{N}$-cadherin $(p<0.05)$ and vimentin $(p<0.01)$ but inhibited expression of E-cadherin $(p<0.01)$ and ZO-1 $(p<0.01)$ in MTC cells in comparison with the NC inhibitor group (Figure $2 \mathrm{D}$ ).

\section{HBEGF was a direct target of miR-376c-3p}

HBEGF was found to be a potential target of miR-376c-3p (Figure $3 \mathrm{~A}$ ) using the TargetScan prediction tool (www.targetscan.org). MiR$376 c-3 p$ mimic dramatically inhibited the luciferase activity of the wild-type HBEGF 3'UTR by $59.46 \%$ in comparison with that of the NC mimic $(p<0.01)$, while miR-376c-3p overexpression ex- 
A
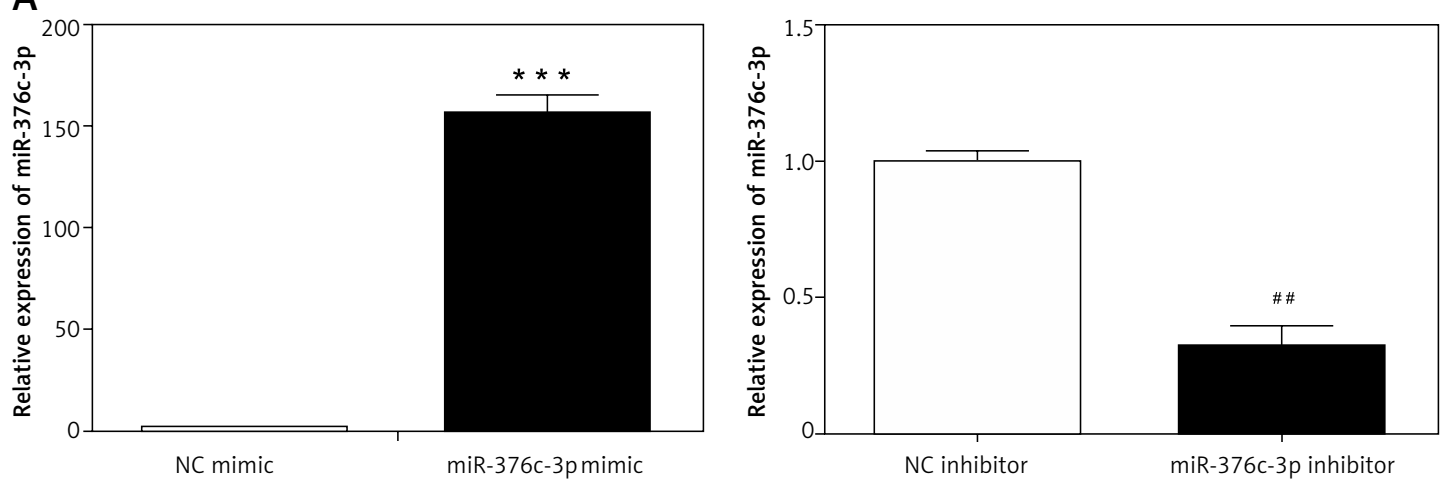

B

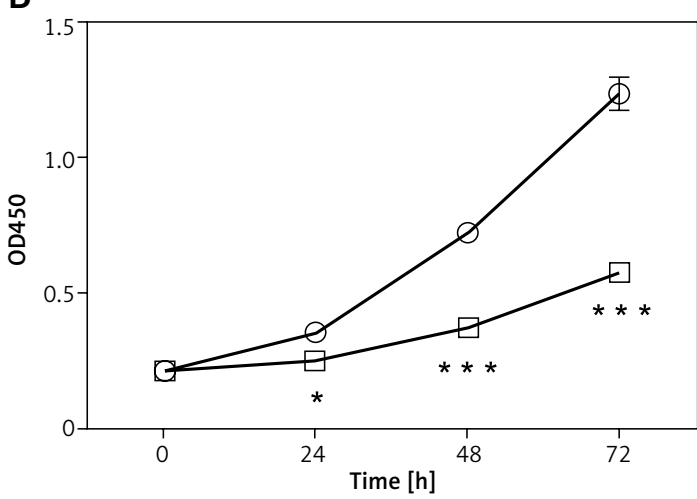

$\rightarrow$ NC mimic $\rightleftarrows$ miR-376c-3pmimic

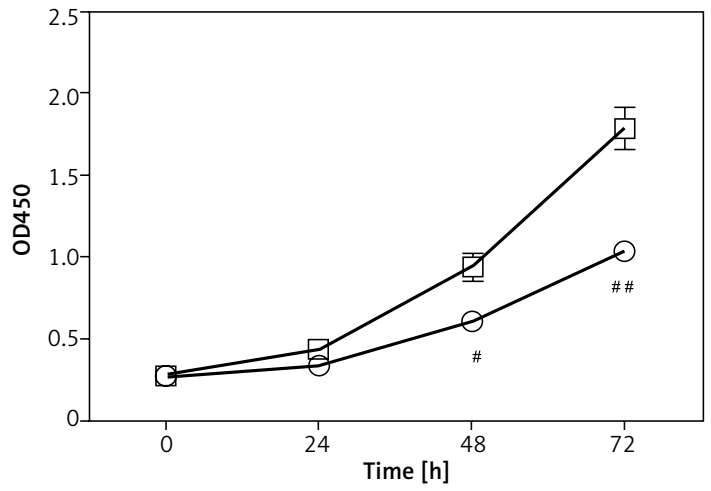

- NC inhibitor $\quad$ miR-376c-3p inhibitor

C

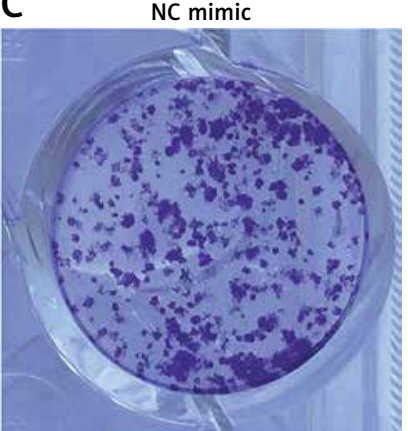

miR-376c-3p mimic
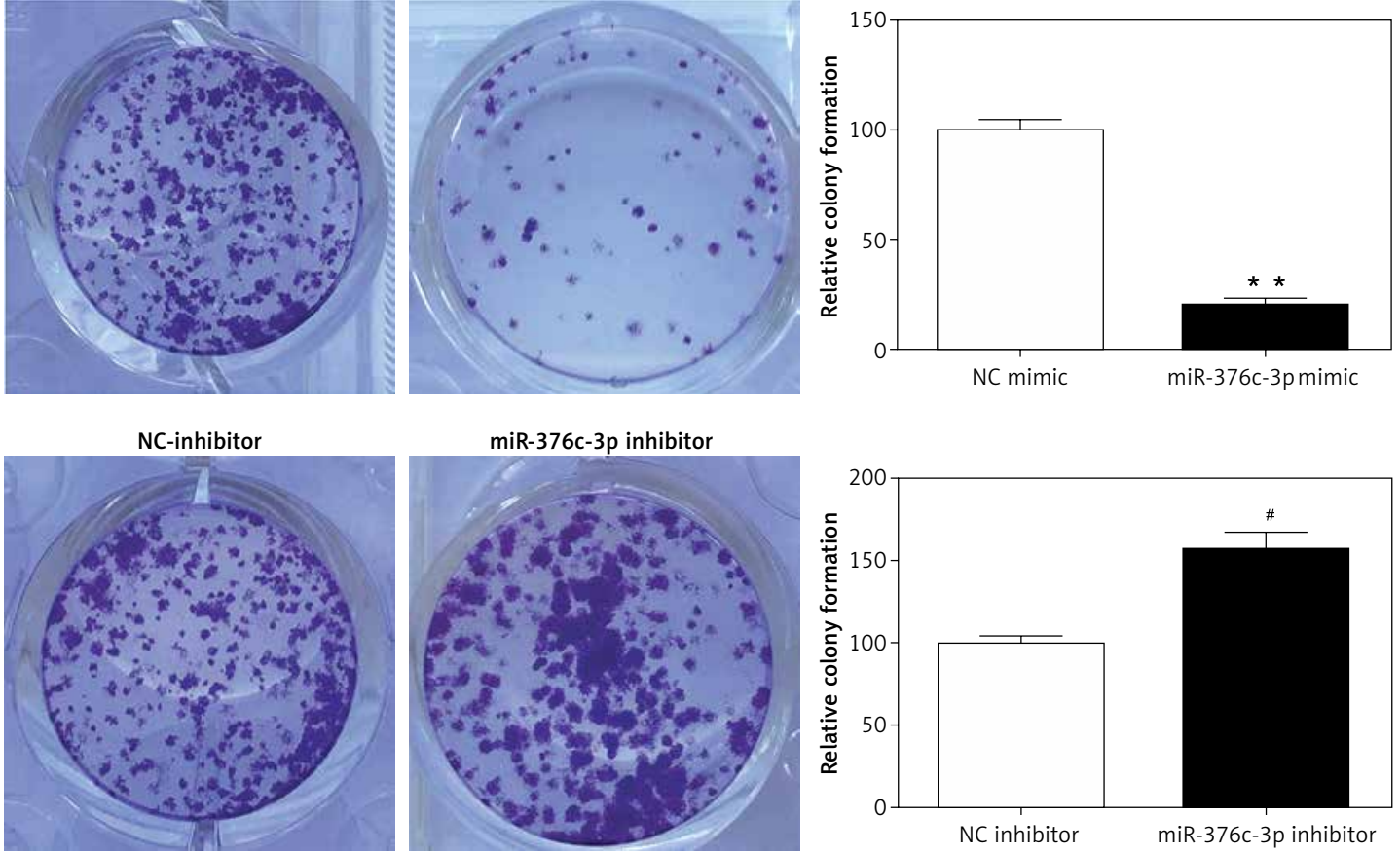

miR-376c-3p inhibitor
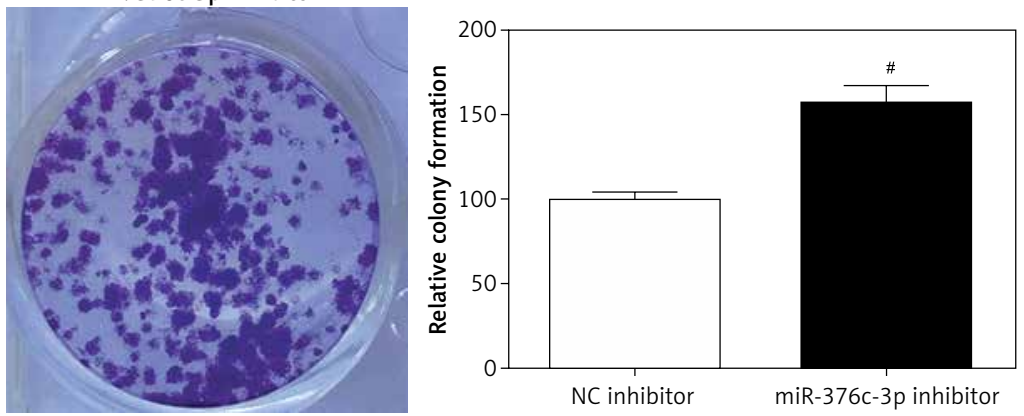

Figure 1. Effect of miR-376c-3p overexpression on MTC cell proliferation. MZ-CRC-1 cells transfected with miR-376c-3p mimic or miR-376c-3p inhibitor. A - Expression level of MiR-376c-3p was analyzed with qPCR. B, C - The proliferation of MTC cells was examined using CCK-8 (B) and soft agar colony formation assay (C)

${ }^{*} p<0.05,{ }^{* *} p<0.01$ and ${ }^{* *} p<0.001$ compared to the NC mimic group. ${ }^{*} p<0.05$ and ${ }^{\# \#} p<0.01$ compared to the NC inhibitor group.

hibited little luciferase activity of mutant HBEGF $3^{\prime} U T R$, suggesting the interruption of the interaction between miR-376c-3p and HBEGF (Figure 3 B).
Further analysis found that miR-376c-3p mimic reduced the expression level of mRNA $(p<0.05)$ (Figure $3 C$ ), and protein $(p<0.01$ ) (Figure 3 D), of 


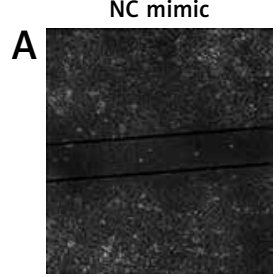

NC inhibitor

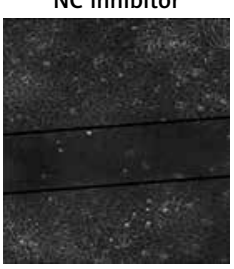

NC mimic

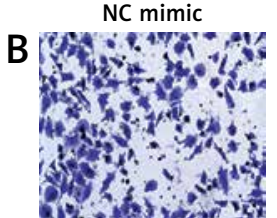

NC inhibitor

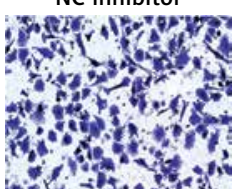

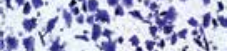

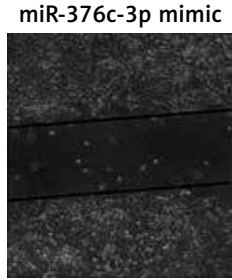

miR-376c-3p inhibitor

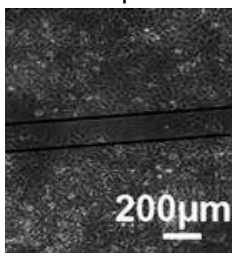

miR-376c-3p mimic
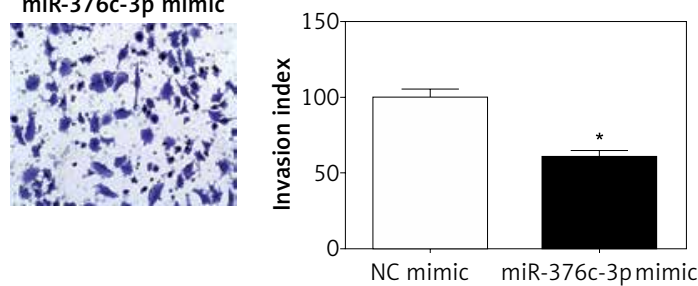

miR-376c-3p inhibitor
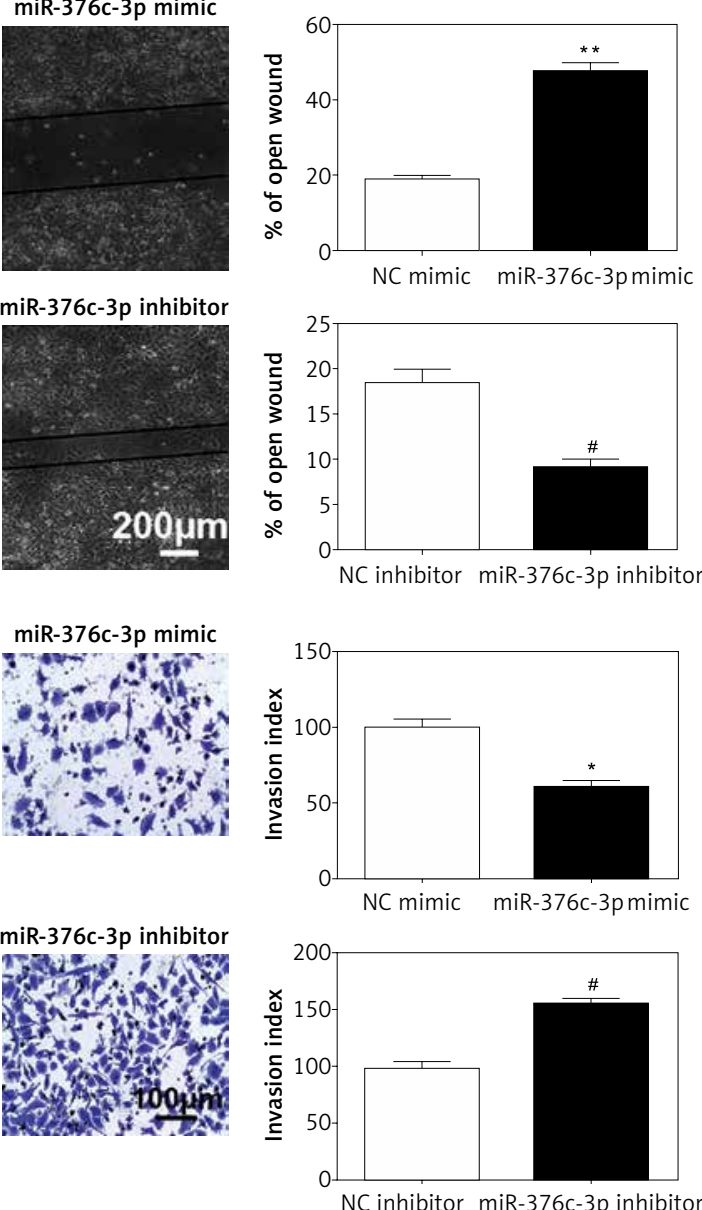

NC inhibitor miR-376c-3p inhibitor

NC inhibitor miR-376c-3p inhibitor
C
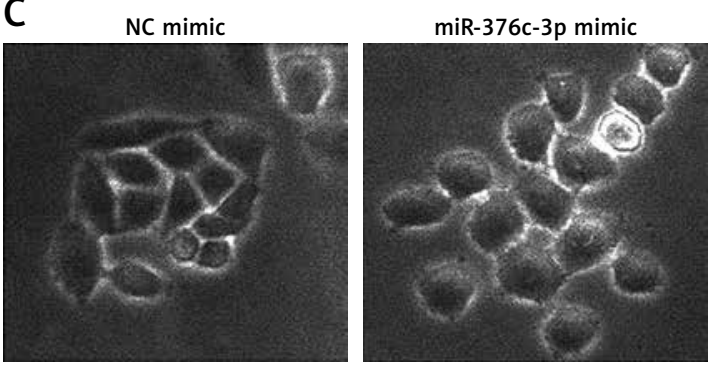

D

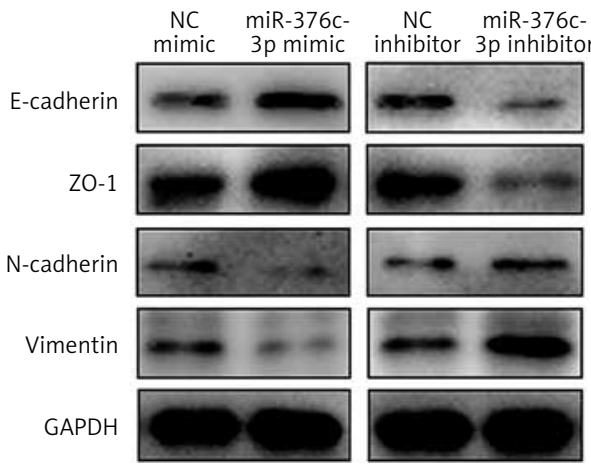

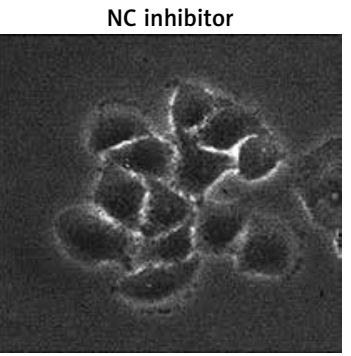

miR-376c-3p inhibitor
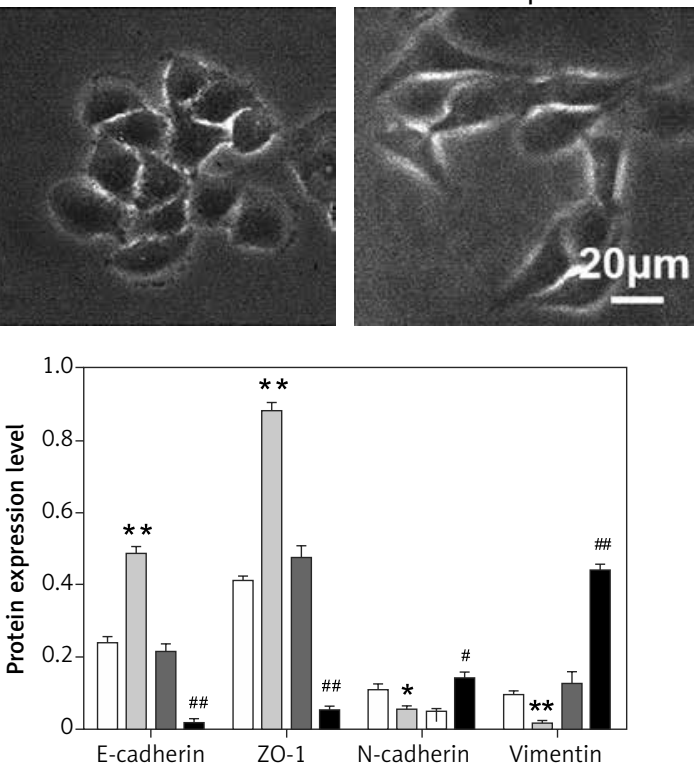

$\square$ NC mimic $\quad \square$ miR-376c-3pmimic

$\square$ NC inhibitor miR-376c-3p inhibitor

Figure 2. Effect of miR-376c-3p overexpression on MTC cell migration and invasion. A - Representative images and quantification of the wound healing assay of MTC cells transfected with miR-376c-3p mimic or miR-376c-3p inhibitor. B - Representative images and quantification of invaded cells using the transwell assay. C - Representative micrographs of aforementioned cell. D - Expression levels of E-cadherin, ZO-1, N-cadherin and vimentin were examined using the western blot. GAPDH was used as the internal control

${ }^{*} p<0.05$ and ${ }^{* *} p<0.01$ compared to NC mimic group. ${ }^{*} p<0.05$ and ${ }^{\# \#} p<0.01$ compared to NC inhibitor group. 
A

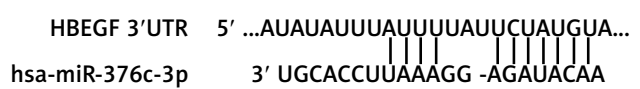

C

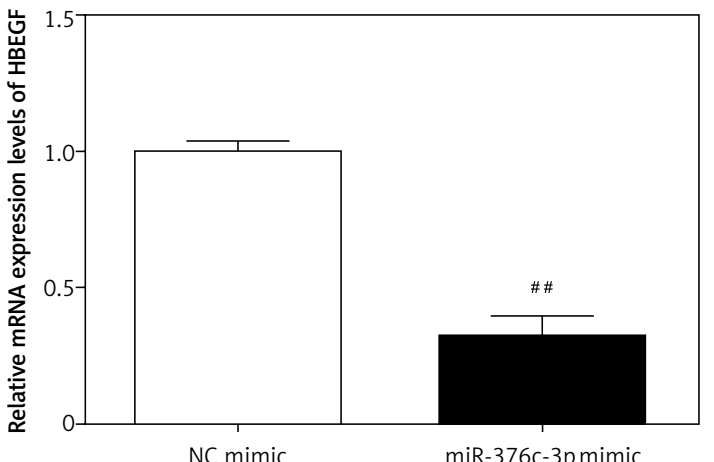

D

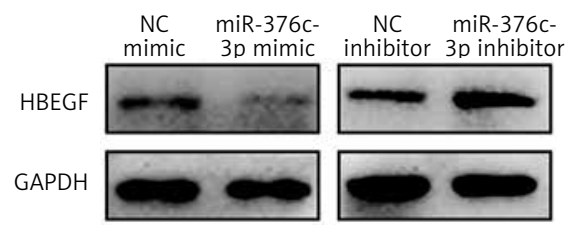

B
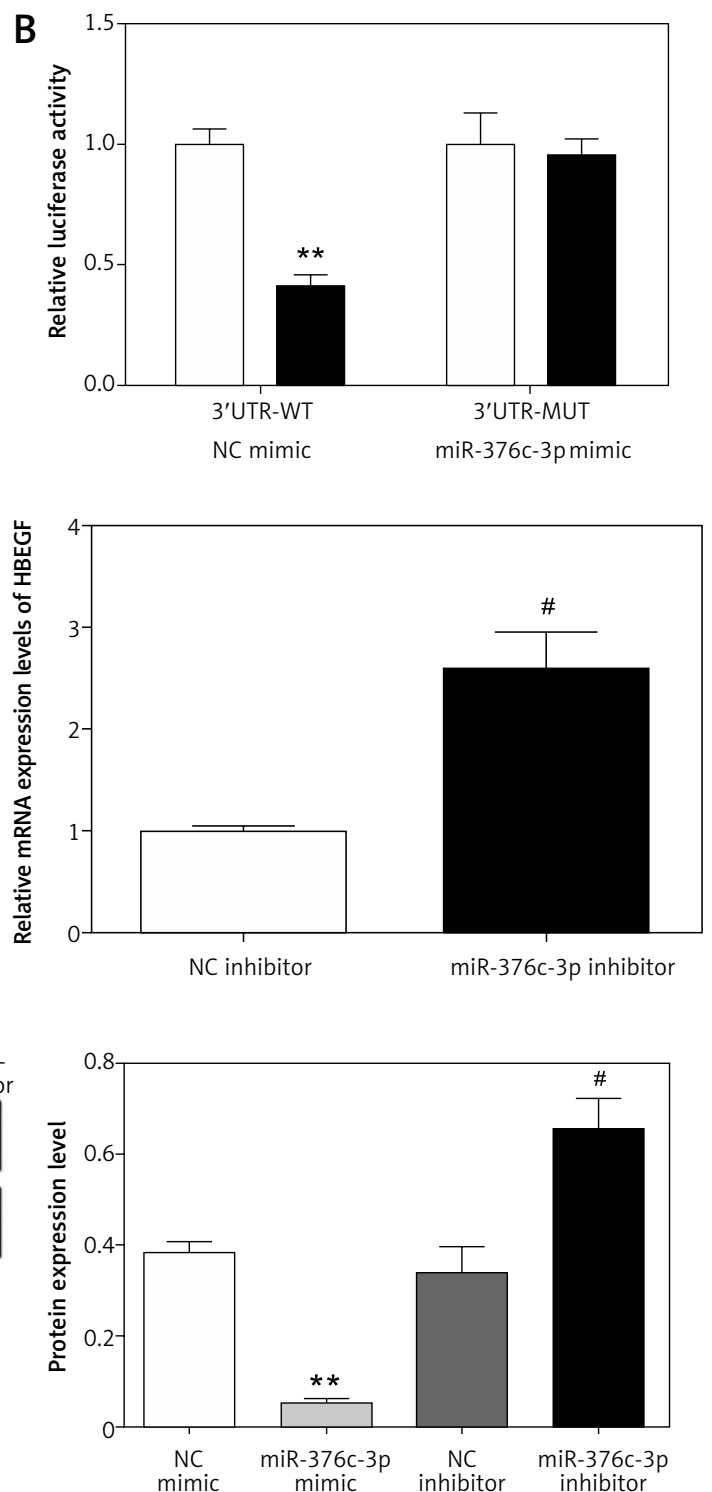

Figure 3. Verification of miR-376c-3p target gene. A - Schematic diagram of miR-376c-3p binding sites of HBEGF 3'UTR. B - The luciferase activity of the plasmid containing wild-type or mutant HBEGF 3'UTR was analyzed in MZCRC-1 cells transfected with NC mimic or miR-376c-3p mimic. C, D - HBEGF mRNA and protein abundance in MTC cells transfected with the miR-376c-3p mimic or miR-376c-3p inhibitor was analyzed using qPCR (C) and western blot (D), respectively

${ }^{*} p<0.05$ and ${ }^{* *} p<0.01$ compared to the NC mimic group. ${ }^{*} p<0.05$ compared to the NC inhibitor group.

HBEGF in MZ-CRC-1 cells, compared to the NC mimic. In contrast, MTC cells transfected with the miR$376 c-3 p$ inhibitor showed higher mRNA $(p<0.05)$ (Figure $3 C$ ) and protein $(p<0.05)$ (Figure 3 D) level of HBEGF than those transfected with NC inhibitor. The results suggested that expression of miR-376c-3p was inversely correlated with that of HBEGF in MZ-CRC-1 cells.

HBEGF overexpression blocked the antitumor roles of miR-376c-3p in MTC cells

MZ-CRC-1 cells were transfected with NC mimic, miR-376c-3p mimic or miR-376c-3p mimic along with the HBEGF overexpression plasmid. MiR-376c$3 p$ mimic inhibited HBEGF expression $(p<0.05)$ (Figure $4 \mathrm{~A}$ ), while co-transfection with HBEGF-overexpression plasmid completely annihilated the inhibition by upregulating miR-376c-3p $(p<0.05)$ (Figure $4 \mathrm{~A})$. The results of CCK-8 assay showed that HBEGF overexpression nullified the inhibitory effect on MTC cell proliferation caused by miR-376c-3p mimic in a time-dependent manner $(p<0.05, p<0.01$ and $p<0.01$ for $24 \mathrm{~h}, 48 \mathrm{~h}$ and $72 \mathrm{~h}$ after transfection, respectively) (Figure $4 \mathrm{~B}$ ). Figure $4 \mathrm{C}$ confirmed that miR-376c-3p overexpression inhibited the colony formation of MTC cells $(p<0.01)$, while HBEGF overex- 

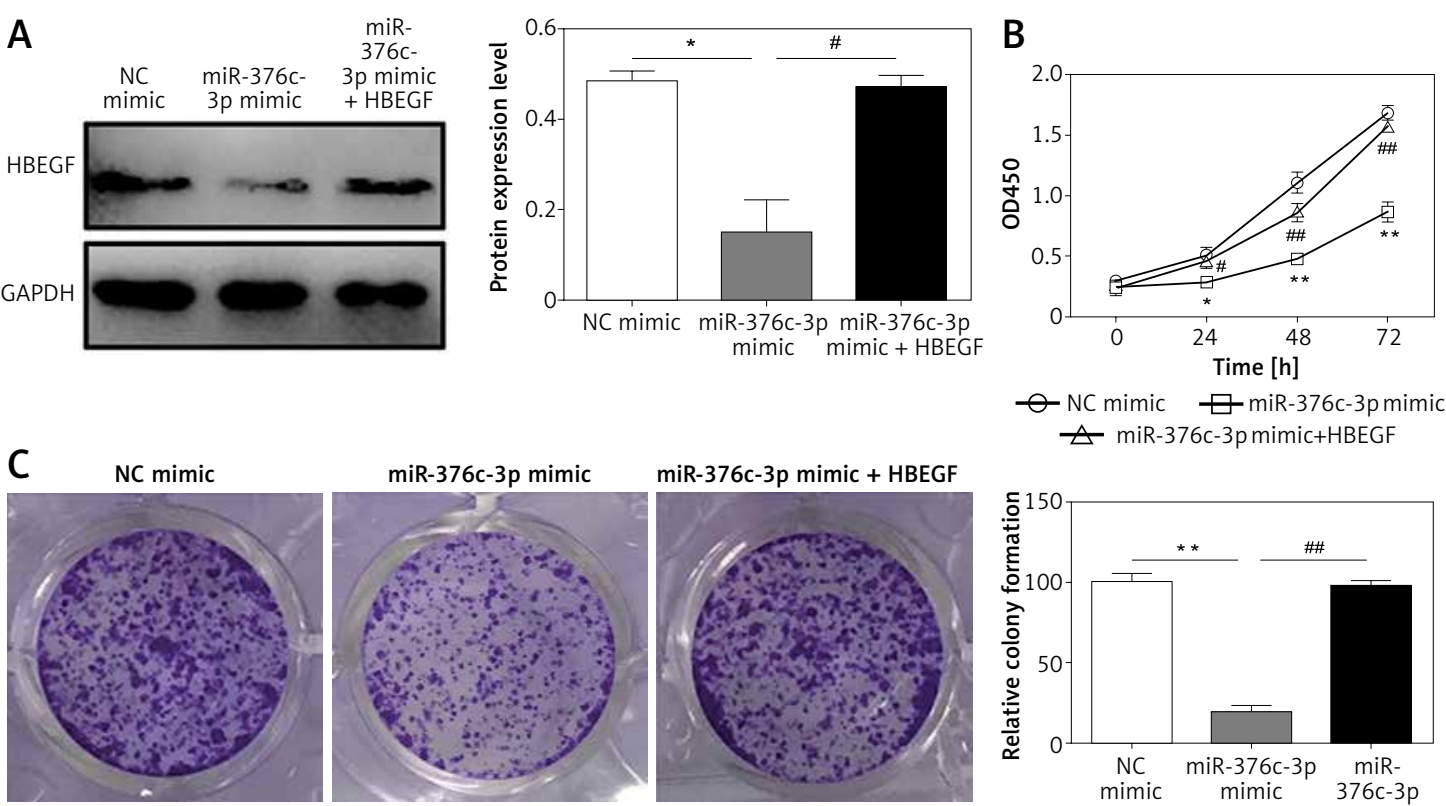

$\vartheta$ NC mimic $\quad$ miR-376c-3pmimic

$\triangle$ miR-376c-3p mimic + HBEGF

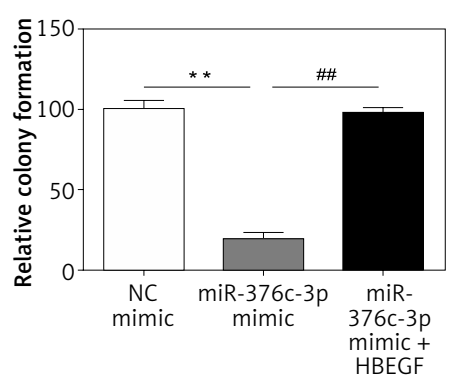

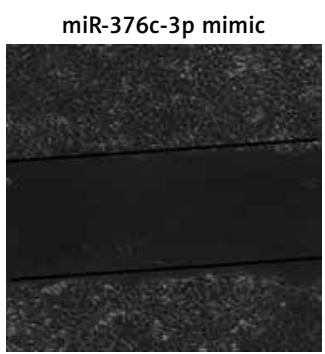
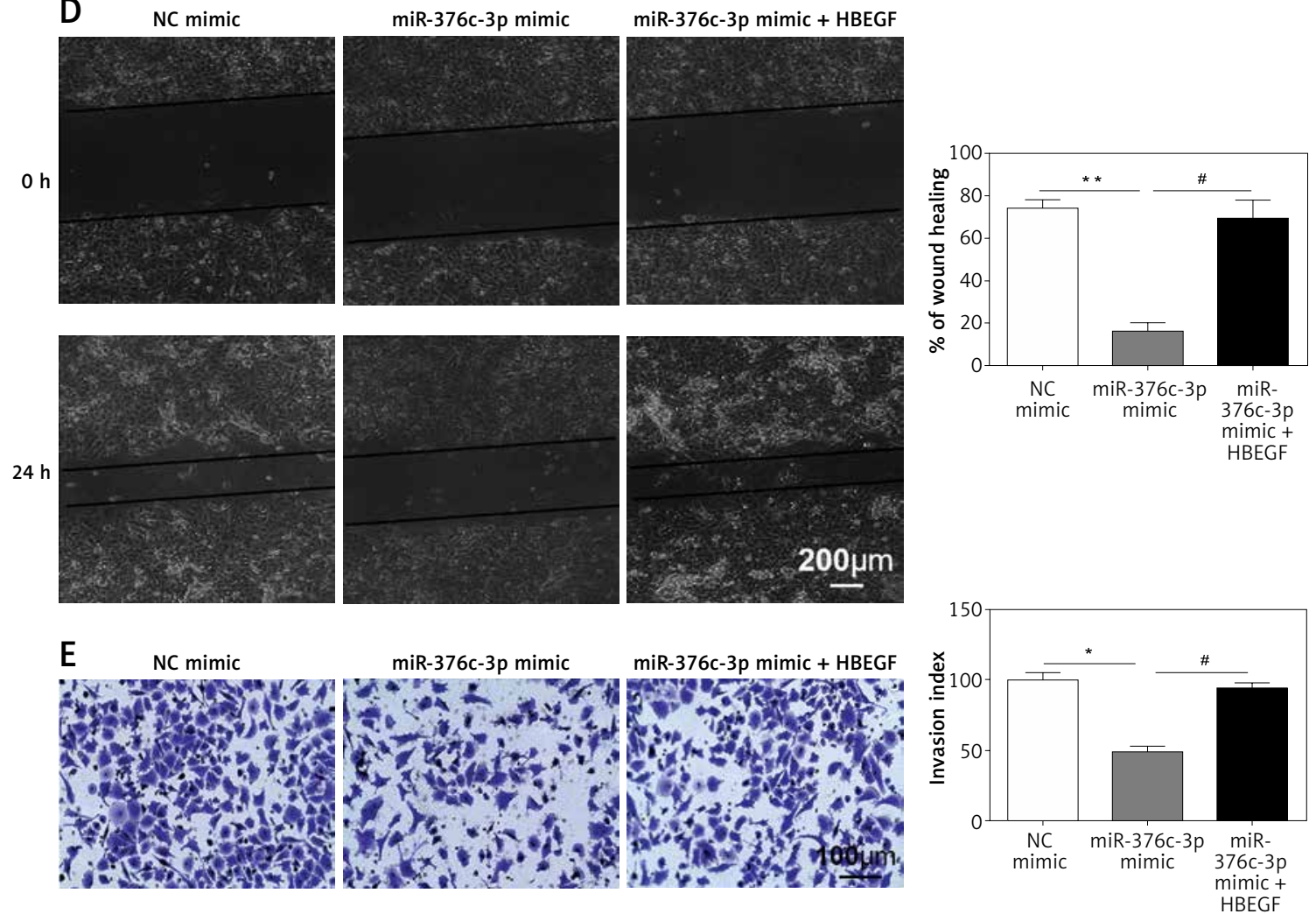

Figure 4. Effects of HBEGF overexpression on proliferation, migration and invasion of MTC cells. MTC cells were transfected with miR-376c-3p mimic or miR-376c-3p mimic plus HBEGF overexpression plasmid. A-HBEGF protein level in indicated cells was analyzed by western blotting. B, C - Proliferation of MZ-CRC-1 cells was determined via CCK-8 (B) and soft agar colony formation analysis (C). D, E - Migration and invasion of MTC cells were determined using wound healing (D) and transwell (E) assay, respectively

${ }^{*} p<0.05$ and ${ }^{* *} p<0.01$ compared to the NC mimic group. ${ }^{*} p<0.05$ and ${ }^{\# \#} p<0.01$ compared to the miR-376c-3p mimic group.

pression annihilated the inhibitory effects on MTC cell proliferation $(p<0.01)$.

On the other hand, the wound healing assay demonstrated that miR-376c-3p mimic reduced the migratory ability of MTC cells to $\sim 20 \%$, compared with the NC mimic ( $p<0.01$ ) (Figure $4 \mathrm{D}$ ); HBEGF overexpression cancelled out such an effect $(p<0.05)$ (Figure 4 D). The transwell assay 
A

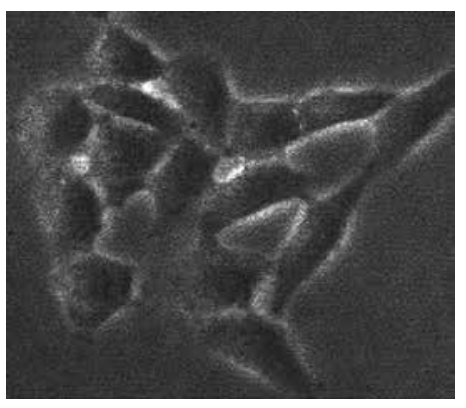

B

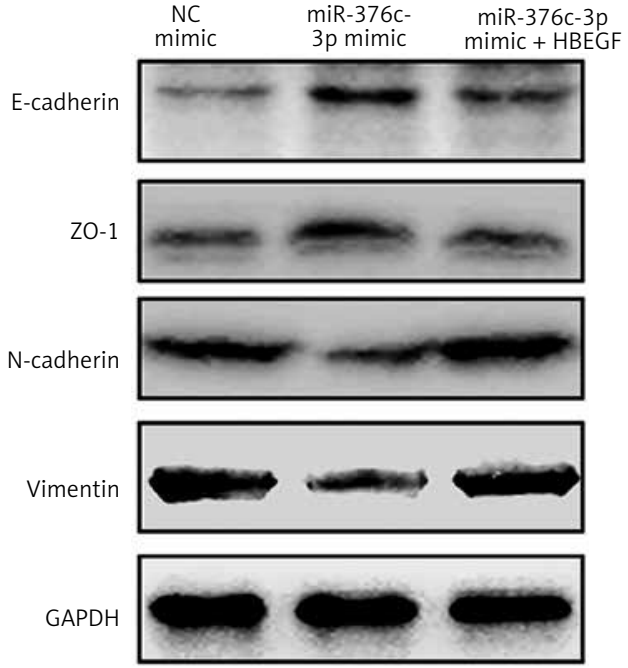

miR-376c-3p mimic

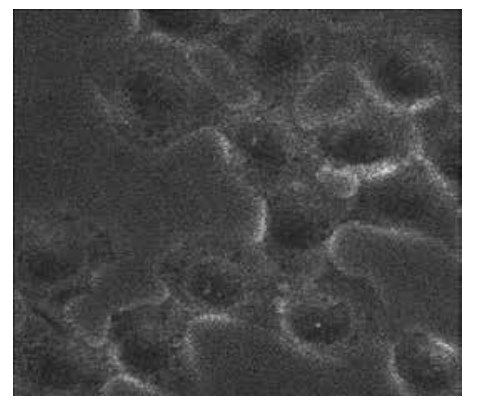

miR-376c-3p mimic + HBEGF

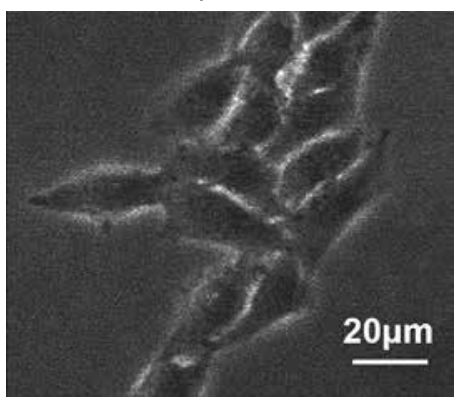

Figure 5. Effect of miR-376c-3p on the EMT of MTC cells was modulated by HBEGF. A - Representative images of indicated cells. B - Western blot analysis of protein levels of E-cadherin, ZO-1, N-cadherin and vimentin. GAPDH was used as the internal control

${ }^{*} p<0.05$ and ${ }^{* *} p<0.01$ compared to the NC mimic group. ${ }^{*} p<0.05$ and ${ }^{\# \#} p<0.01$ compared to the miR-376c-3p mimic group.

confirmed that HBEGF overexpression removed the miR-376c-3p-induced inhibition of the invasion of MTC cells $(p<0.05)$ (Figure $4 \mathrm{E})$.

\section{MiR-376c-3p regulated EMT by targeting HBEGF}

The relationship between EMT and tumor dissemination has been well studied $[16,17]$. MZCRC- 1 cells of the miR-376c-3p mimic group showed weaker invasion ability. Cells transfected with the miR-376c-3p mimic plus HBEGF overexpression plasmid displayed an elongated and spindle-like shape (Figure $5 \mathrm{~A}$ ). Western blot results demonstrated that miR-376c-3p mimic downregulated $\mathrm{N}$-cadherin $(p<0.01)$ and vimentin $(p<0.01)$ but upregulated E-cadherin $(p<0.001)$ and ZO-1 ( $p<$ 0.01 ) in MTC cells (Figure 5 B). However, the effects were then annihilated upon HBEGF overexpression $(p<0.01)$ (Figure 5 B).

\section{Discussion}

Lymph node metastasis occurred in $70 \%$ patients diagnosed with MTC and distant metastasis in 10\%
[18-20]. Although some clinical therapies, such as surgery and radiation therapy, have been used for treating MTC, regional recurrence or distant metastasis remain the main challenge of currently available MTC therapies. It is generally believed that $75 \%$ of MTCs occur as sporadic and $25 \%$ as hereditary type, which is also known as multiple endocrine neoplasia 2 (MEN2) [13]. Most MEN2 syndrome cases and about half of sporadic MTCs are caused by RET mutations [2, 9]. The MZ-CRC-1 cells with mutation of $R E T^{M 918 T}$ were hence used for our study.

Multiple miRNAs were dysregulated in MTC and were closely correlated with progression, metastasis and invasion of MTC. Galuppini et al. found that expression of miR-375 was proportional to the size of the neoplasm [18]. Spitschak et al. recently demonstrated that miR182 was crucial to the manifestation of aggressiveness of MTC [2]. Hudson et al. showed that the upregulation of miR-375 and miR-10a was associated with the development of MTC [21].

MiR-376c-3p is often down-regulated in various tumors, such as colorectal cancer [11], HNSCC [12] and oral squamous cancer [13], exhibiting can- 
cer-protective effects. In this study it was found that miR-376c-3p overexpression exhibited tumor inhibitory effects, as shown by significant suppression of the proliferation, migration and invasion of MZ-CRC-1 cells. In contrast, downregulating miR$376 c-3 p$ through miRNA inhibitor regained tumor promoting activities, with remarkable promotion of the proliferation and aggressiveness of MTC cells.

It is generally accepted that miRNA may function as a tumor suppressor in various tumors via regulating the expression of its target genes [22]. Recently, two direct targets of miR-376c-3p, HOXB7 in oral squamous cancer [13] and LINC00152 in colorectal cancer [11], have been identified. In this study, bioinformatics analysis showed that there were potential miR-376c-3p binding sites within the HBEGF 3'UTR. Luciferase activity assay results demonstrated that miR-376c-3p mimic suppressed the luciferase activity of wild-type HBEGF 3'UTR, suggesting that HBEGF was a direct downstream target of miR-376c-3p in MTC cells. Furthermore, HBEGF expression was significantly downregulated in MTC cells in the miR-376c-3p mimic group, indicating that miR-376c-3p could negatively regulate the expression of HBEGF in MTC cells.

HBEGF has been proved to be critical in tumorigenesis, progression and metastasis of different solid tumors [23-25]. Previous studies showed that HBEGF was up-regulated in thyroid cancer [26] and promoted the invasion and metastasis of thyroid cancer cells $[26,27]$. It was hence speculated that miR-376c-3p might inhibit MTC by downregulating HBEGF. To our delight, the in vitro results showed that HBEGF overexpression annihilated the antitumor effects of miR-376c-3p mimic transfection on the MTC cells, as shown by the significant increase in the growth, migration and invasion of the MTC cells.

The EMT is known to be involved in tumor invasion and metastasis $[28,29]$. It is also a key pathologic pathway for the development of MTC [30]. Previous studies showed that decrease in expression of epithelial markers (E-cadherin, ZO-1) and increase in expression of mesenchymal markers (N-cadherin, vimentin) induced mesenchymal phenotype and subsequently stimulated migration and metastasis of tumor cells [31-33]. It has also been reported that HBEGF upregulation promoted the EMT ability of various cancers, such as lung cancer [34], ovarian cancer [35] and pancreatic carcinoma [36]. In order to gain a deeper insight into the molecular mechanisms underlying the anti-metastasis role of miR-376c-3p, expression of EMT markers in MTC cells was hence examined. The results showed that miR-376c-3p mimic inhibited EMT phenotype by inhibiting the expression of $\mathrm{N}$-cadherin and vimentin and by upregulating that of E-cadherin and ZO-1. HBEGF overexpres- sion significantly increased expression N-cadherin and vimentin but downregulated E-cadherin and ZO-1, thus stimulating the EMT in MTC cells - the consequence of which was consistent with that caused by the miR-376c-3p inhibitor. The results suggested that miR-376c-3p negatively regulated HBEGF expression, modulated the expression of EMT markers and exhibited anti-metastasis effects on MTC cells.

In conclusion, our study demonstrated that the downregulation of miR-376c-3p and upregulation of HBEGF in MZ-CRC-1 cells were closely related to the development and metastasis of MTC. MiR$376 c-3 p$ functioned as a tumor suppressor in MTC cells by directly binding and negatively modulating HBEGF. These findings suggested that miR$376 c-3 p$ and HBEGF could be used as potential therapeutic targets for the treatment of MTC.

Our current study suggested that miR-376c-3p directly bound to HBEGF 3'UTR and downregulated HBEGF, which subsequently suppressed the growth, migration and invasion of $M Z-C R C-1$ cells.

\section{Acknowledgments}

This work was supported by a project of Nanjing First Hospital, Nanjing Medical University.

\section{Conflict of interest}

The authors declare no conflict of interest.

\section{References}

1. Wells SA Jr., Asa SL, Dralle H, et al. Revised American Thyroid Association guidelines for the management of medullary thyroid carcinoma. Thyroid 2015; 25: 567-610.

2. Spitschak A, Meier C, Kowtharapu B, Engelmann D, Putzer BM. MiR-182 promotes cancer invasion by linking RET oncogene activated NF-kappaB to loss of the HES1/ Notch1 regulatory circuit. Mol Cancer 2017; 16: 24.

3. de Groot JW, Plaza Menacho I, Schepers H, et al. Cellular effects of imatinib on medullary thyroid cancer cells harboring multiple endocrine neoplasia type $2 \mathrm{~A}$ and $2 \mathrm{~B}$ associated RET mutations. Surgery 2006; 139: 806-14.

4. Ferreira CV, Siqueira DR, Ceolin L, Maia AL. Advanced medullary thyroid cancer: pathophysiology and management. Cancer Manag Res 2013; 5: 57-66.

5. Chau NG, Haddad RI. Vandetanib for the treatment of medullary thyroid cancer. Clin Cancer Res 2013; 19: 524-9.

6. Bartel DP. MicroRNAs: genomics, biogenesis, mechanism, and function. Cell 2004; 116: 281-97.

7. Farh KK, Grimson A, Jan C, et al. The widespread impact of mammalian microRNAs on mRNA repression and evolution. Science 2005; 310: 1817-21.

8. Li N, Fu H, Tie $\mathrm{Y}$, et al. miR-34a inhibits migration and invasion by down-regulation of c-Met expression in human hepatocellular carcinoma cells. Cancer Lett 2009; 275: 44-53.

9. Ye Y, Zhuang J, Wang G, He S, Ni J, Xia W. MicroRNA-139 targets fibronectin 1 to inhibit papillary thyroid carcinoma progression. Oncol Lett 2017; 14: 7799-806.

10. Cheng L, Zhou R, Chen M, Feng L, Li H. MicroRNA-150 targets Rho-associated protein kinase 1 to inhibit cell 
proliferation, migration and invasion in papillary thyroid carcinoma. Mol Med Rep 2017; 16: 2217-24.

11. Zhang YH, Fu J, Zhang ZJ, Ge CC, Yi Y. LncRNA-LINC00152 down-regulated by miR-376c-3p restricts viability and promotes apoptosis of colorectal cancer cells. Am J Trans Res 2016; 8: 5286-97.

12. Chang WM, Lin YF, Su CY, et al. Dysregulation of RUNX2 activin-A axis upon miR-376c downregulation promotes lymph node metastasis in head and neck squamous cell carcinoma. Cancer Res 2016; 76: 7140-50.

13. Wang K, Jin J, Ma T, Zhai H. MiR-376c-3p regulates the proliferation, invasion, migration, cell cycle and apoptosis of human oral squamous cancer cells by suppressing HOXB7. Biomed Pharmacother 2017; 91: 517-25.

14. Yang R, Fang W, Liang J, et al. Apelin/APJ axis improves angiotensin II-induced endothelial cell senescence through AMPK/SIRT1 signaling pathway. Arch Med Sci 2018; 14: 725-34

15. Ke X, Ke B, Wang X, Wu S, Yang R, Hu C. Additive effects of atorvastatin combined with sitagliptin on rats with myocardial infarction: a pilot study. Arch Med Sci 2017; 13: 956-61.

16. Mani SA, Guo W, Liao MJ, et al. The epithelial-mesenchymal transition generates cells with properties of stem cells. Cell 2008; 133: 704-15.

17. Gunasinghe NP, Wells A, Thompson EW, Hugo HJ. Mesenchymal-epithelial transition (MET) as a mechanism for metastatic colonisation in breast cancer. Cancer Metastasis Rev 2012; 31: 469-78.

18. Galuppini F, Bertazza L, Barollo S, et al. MiR-375 and YAP1 expression profiling in medullary thyroid carcinoma and their correlation with clinical-pathological features and outcome. Virchows Arch 2017; 471: 651-8.

19. Lin SF, Lin JD, Hsueh C, Chou TC, Wong RJ. Activity of roniciclib in medullary thyroid cancer. Oncotarget 2018; 9: 28030-41.

20. Moley JF. Medullary thyroid carcinoma: management of lymph node metastases. J Natl Compr Canc Netw 2010; 8: 549-56.

21. Hudson J, Duncavage E, Tamburrino A, et al. Overexpression of miR-10a and miR-375 and downregulation of YAP1 in medullary thyroid carcinoma. Exp Mol Pathol 2013; 95: 62-7.

22. Rang Z, Yang G, Wang YW, Cui F. miR-542-3p suppresses invasion and metastasis by targeting the proto-oncogene serine/threonine protein kinase, PIM1, in melanoma. Biochem Biophys Res Commun 2016; 474: 315-20.

23. Shin $\mathrm{CH}$, Robinson JP, Sonnen JA, et al. HBEGF promotes gliomagenesis in the context of Ink4a/Arf and Pten loss. Oncogene 2017; 36: 4610-8.

24. Wei LQ, Liang HT, Qin DC, Jin HF, Zhao Y, She MC. MiR-212 exerts suppressive effect on SKOV3 ovarian cancer cells through targeting HBEGF. Tumour Biol 2014; 35: 12427-34.

25. Zhou ZN, Sharma VP, Beaty BT, et al. Autocrine HBEGF expression promotes breast cancer intravasation, metastasis and macrophage-independent invasion in vivo. Oncogene 2014; 33: 3784-93.

26. Ria R, Simeon V, Melaccio A, et al. Gene expression profiling of normal thyroid tissue from patients with thyroid carcinoma. Oncotarget 2016; 7: 29677-88.

27. Ota I, Higashiyama S, Masui T, Yane K, Hosoi H, Matsuura N. Heparin-binding EGF-like growth factor enhances the activity of invasion and metastasis in thyroid cancer cells. Oncol Rep 2013; 30: 1593-600.
28. Lee Y, Jung WH, Koo JS. Adipocytes can induce epithelial-mesenchymal transition in breast cancer cells. Breast Cancer Res Treat 2015; 153: 323-35.

29. Thiery JP. Epithelial-mesenchymal transitions in tumour progression. Nat Rev Cancer 2002; 2: 442-54.

30. Lian EY, Maritan SM, Cockburn JG, et al. Differential roles of RET isoforms in medullary and papillary thyroid carcinomas. Endocr Relat Cancer 2017; 24: 53-69.

31. Dong P, Xiong Y, Watari $\mathrm{H}$, et al. MiR-137 and miR-34a directly target Snail and inhibit EMT, invasion and sphere-forming ability of ovarian cancer cells. J Exp Clin Cancer Res 2016; 35: 132.

32. Lee JM, Dedhar S, Kalluri R, Thompson EW. The epithelial-mesenchymal transition: new insights in signaling, development, and disease. J Cell Biol 2006; 172: 973-81.

33. Zhang HW, Wang EW, Li LX, et al. A regulatory loop involving miR-29c and Sp1 elevates the TGF-beta1 mediated epithelial-to-mesenchymal transition in lung cancer. Oncotarget 2016; 7: 85905-16.

34. Kuo PL, Huang MS, Cheng DE, Hung JY, Yang CJ, Chou SH. Lung cancer-derived galectin-1 enhances tumorigenic potentiation of tumor-associated dendritic cells by expressing heparin-binding EGF-like growth factor. J Biol Chem 2012; 287: 9753-64.

35. Yagi H, Yotsumoto F, Miyamoto S. Heparin-binding epidermal growth factor-like growth factor promotes transcoelomic metastasis in ovarian cancer through epithelial-mesenchymal transition. Mol Cancer Ther 2008; 7: 3441-51

36. Wang F, Sloss C, Zhang X, Lee SW, Cusack JC. Membrane-bound heparin-binding epidermal growth factor like growth factor regulates E-cadherin expression in pancreatic carcinoma cells. Cancer Res 2007; 67: 8486-93. 